\title{
The Organization of Stepping in Patients with Parkinson's Disease: Bradykinesia or Discoordination?
}

\author{
Anne Beuter, Lise Carrière, Bradford McFadyen and Serge Gauthier
}

\begin{abstract}
The purpose of this experiment was to quantify and analyse multijoint coordination of patients with Parkinson's disease $(\mathrm{N}=5)$ and control subjects $(\mathrm{N}=5)$ during forward and backward stepping motions executed at different cadences. Coordinates of reflective markers placed on the shoulder, hip, knee, ankle and metatarsal joints were recorded in the sagittal plane using a video motion analysis system. Kinematic and kinetic analyses provided angular displacements and velocities as well as joint moments of force and powers. Results suggest the presence of two types of discoordination: one type is velocity-dependent, hence related to bradykinesia, whereas the other type appears to reflect qualitatively different coordination patterns.
\end{abstract}

RÉSUMÉ: L’organisation du mouvement pour exécuter des pas chez les parkinsoniens: bradykinésie ou incoordination. L'objectif de cette étude était de quantifier et d'analyser la coordination multiarticulaire de patients atteints de la maladie de Parkinson $(\mathrm{N}=5)$ et de sujets contrôles $(\mathrm{N}=5)$ pendant des mouvements d'enjambement effectués vers l'avant et l'arrière à différentes cadences. Les coordonnées de marqueurs réfléchissants placés sur l'épaule, la hanche, le genou, la cheville et le métatarse ont été enregistrées dans le plan sagittal à l'aide d'un système vidéo informatisé d'analyse du mouvement. Les analyses cinématiques et cinétiques ont permis de quantifier les déplacements et vitesses angulaires ainsi que les moments de force et la puissance au niveau des différentes articulations. Les résultats suggèrent la présence de deux types d'incoordination chez ces patients: l'un dépend de la vitesse d'exécution du mouvement et est relié à la bradykinésie, alors que l'autre semble correspondre à des patrons de coordination qualitativement différents.

Can.J. Neurol. Sci. 1992; 19: 8-16

A number of studies have recently pointed out that past objective tests of movement deficits in patients with Parkinson's disease (PD) have focused on a variety of isolated motor tasks which had little to do with the activity of everyday life. ${ }^{1.2}$ The results of studies looking at simple movements in patients with PD were generally variable and the performance of these patients looked relatively normal despite clear evidence of advanced neurological disease. ${ }^{2-6}$ Recently, Johnels et al. ${ }^{2}$ have stressed the importance of using comprehensive, objective and reproducible quantitative methods to assess the degree of disability rather than the severity of isolated symptoms.

One way to assess the degree of disability of neurological patients is to quantify and analyse movement coordination during sequences of multiarticulate movements belonging to our everyday behavioural repertoire. For example, Johnels et al. ${ }^{2}$ measured the degree of disability of $\mathrm{PD}$ by recording body movements in a posturo-locomotor-manual test executed at maximum speed. Quantitative profiles of specific movement disabilities (posture, locomotion and manipulation) were generated. In Johnels' study however, a direct comparison between the coordination patterns of control subjects and patients with PD was not possible since patients with PD were up to 13.6 times slower than control subjects. To our knowledge, past studies on movement coordination in patients with PD have not taken into consideration the fact that patients with PD may move slower in addition to using qualitatively different patterns of coordination. Few studies have analysed the coordination impairments of patients with PD in multijoint movements. ${ }^{7}$ Two exceptions have examined locomotion and psychomotor performance. ${ }^{6,8}$

Recently, claims that patients with PD use qualitatively different coordination patterns have been forwarded. For example, in a study focusing on the coordination of well-learned speech movements, Connor et al. ${ }^{1}$ examined the relative contribution of

From the Département de Kinanthropologie, Université du Québec à Montréal (A.B., L.C., B.M.), Centre for Nonlinear Dynamics in Physiology \& Medicine (McGill) (A.B.) and McGill Center for Studies in Aging (S.G.)

Received June 11, 1991. Accepted in final form August 14, 1991

Reprint requests to: Dr. Anne Beuter, Département de Kinanthropologie, Université du Québec à Montréal, CP 8888, Suc A, Montréal, Québec, Canada H3C 3P8 
upper lip, lower lip and jaw to the total oral closing displacements and found that patients with PD used significantly more lower lip movement than control subjects. In normal subjects this pattern is highly stereotyped and these results suggest that patients with PD use different coordination patterns.

Interpreting differences in coordination patterns is not easy since they may represent both primary or secondary symptoms of the disease. For example, Sheridan and Flowers ${ }^{9}$ have suggested that PD can cause greater inherent variability in the endpoints when patients make discrete aiming movements from a peripheral starting position to a target line located in front of them. To execute this task accurately, patients with PD must (1) move more slowly, (2) use visual guidance and (3) make small amplitude movements. In this example, the changes in coordination related to a greater variability may correspond to a primary symptom of the disease while moving slowly, using visual guidance, making small amplitude movements may represent secondary symptoms or behavioural adaptations to the disease. These behavioural adaptations may account for bradykinesia. A detailed analysis of a complex movement belonging to our everyday behavioural repertoire could provide a way to differentiate bradykinesia from discoordination when movement duration is taken into account and bring new insights into the role of the basal ganglia in motor control.

Thus, the aim of this study was to examine coordination impairments caused by PD, during stepping motions performed at different cadences. Since it has been suggested that patients with PD have difficulty switching between different movement segments in complex movements, ${ }^{10}$ we quantified and analysed the coordination patterns of patients with $\mathrm{PD}$ during a repetitive movement sequence (stepping forward and stepping backward). Although stepping forward and backward are similar movement segments, one is more frequently used (i.e., stepping forward) while this other is rarely used (i.e., stepping backward) in activity of everyday life.

Coordination patterns were analysed using kinematic and kinetic analyses. Kinematic analysis provided spatial and temporal information at the hip, knee and ankle joints while kinetic analysis provided detailed information about net muscular involvement at each joint and gave a better understanding of the articular moments of force and mechanical powers produced at each joint.

\section{Methodology}

\section{Subjects}

Five males with PD diagnosed at stage III or IV in the classification of Hoehn and $\mathrm{Yahr}^{11}$ agreed to participate in this study. Their ages ranged from 58 to 75 years with a mean of 70 years. The mean body mass was $82.6 \mathrm{~kg}$. The height ranged from 1.70 $\mathrm{m}$ to $1.85 \mathrm{~m}$ with a mean of $1.79 \mathrm{~m}$. When asked to fill out a short questionnaire, four of the subjects indicated, on a four point Likert scale, that they were not anxious or nervous at all before testing, while one said that he was a little nervous or anxious. All patients with PD took some medication including one or more the following: Prolopa, Sinemet, Artane, Symmetrel, Ativan or Benadryl. Varying dosages had been taken from one to seven hours before the experiment. Finally, all patients with PD reported occasional difficulties in walking or stepping and three out of five reported occasional falls.
Five healthy males ranging in age from 59 to 72 years with a mean of 67 years also participated in the experiment as a control group. The mean mass was $73.2 \mathrm{~kg}$ and the height ranged from $1.60 \mathrm{~m}$ to $1.70 \mathrm{~m}$ with a mean of $1.66 \mathrm{~m}$. On the short questionnaire, one subject (N2) reported occasional difficulty in walking or stepping.

\section{Tasks}

While standing with their feet together, the contours of each subject's feet were traced on the floor. Subjects were then instructed to step back and forth five times at varying cadences over a small obstacle $(10.7 \mathrm{~cm}$ high by $2.7 \mathrm{~cm}$ wide) aligned with the centre of the supporting foot while holding onto a chair placed on the supporting side. All patients with PD except one (P3) used their left leg to step over the obstacle while all healthy control subjects preferred to use their right leg. Each subject completed nine series of uninterrupted stepping motions in the following conditions: natural cadence ( 3 times), fast, very fast, slow, very slow, (1 time each) and progressively accelerated (2 times). A total of 45 forward and backward stepping motions were videotaped and analysed. The order of presentation of the fast and slow cadence conditions was counterbalanced between subjects. The two progressively accelerated conditions were executed last. The three natural cadence conditions were recorded at the beginning, middle and end of the testing session.

\section{Kinematic Analysis}

The stepping motions were recorded in the sagittal plane after reflective markers $(2.5 \mathrm{~cm}$ in diameter) were secured on the acromion, greater trochanter, lateral knee condyle, lateral malleolus, head of the fifth metatarsal, and on the obstacle. The subjects were videotaped with an RCA solid state camera at 60 frames/s. The video signal was digitized providing two dimensional coordinates of each marker. After using a nine-point Tukey window algorithm to smooth the data, the relative joint angular displacements were calculated using a convention of 180 degrees corresponding to the straight line alignment of adjacent segments and flexion indicated by values less than 180 . Angular data were then numerically differentiated to obtain joint angular velocities.

Phase shifts (in seconds) between hip and knee joints were also calculated. They correspond to the timing difference in reaching maximum flexion at the hip and knee joints. In the present study, phase shifts were calculated by subtracting the time at which maximum hip flexion was reached from the corresponding value at the knee joint.

For analysis purposes, each condition was divided into five forward and five backward stepping motions using initiation of knee flexion as a criterion. The first forward and the last backward motions were excluded from the analysis to remove the possible influence of movement initiation and termination on the results.

\section{Kinetic Analysis}

Classical link segment analysis ${ }^{12}$ was performed to calculate the joint reaction forces and moments of force at each joint during the swing phase of three different experimental conditions, including natural, very fast and very slow cadences. Swing was defined from the point of elevation of the metatarsal marker greater than $0.5 \mathrm{~cm}$ from the floor (foot lift-off) to when the 
same marker came during its descent within $0.5 \mathrm{~cm}$ of the floor (foot contact). This presents a slightly different cycle than used for the joint kinematics, but was necessary in the absence of a force platform needed to provide a measure of ground reaction forces after landing.

Joint moments of force may be interpreted as an indication of net muscular activity about the joint. In the present sagittal plane analysis, the moment of force indicates the dominant muscle group (i.e., flexor or extensor) contributing to the movement. The net rate at which all muscles about a joint absorb or generate energy is revealed in the mechanical power calculated as the product of the joint moment of force and the relative joint angular velocity. This relationship is revealed graphically in Figure 1 for one trial of forward stepping for a control subject. Positive bursts in the mechanical power curves arise when the moment of force and angular velocity data have the same polarity and are interpreted as energy generation. Negative bursts arise when the predominant moment about the joint counters the angular velocity (that is opposite to the movement) representing energy absorption. In brief, generation of energy assists the movement while absorption of energy counteracts the movement. Such data are useful in interpreting the mechanical strategies used by subjects to perform various dynamic movements such as obstacle

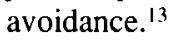

To make within and between subjects comparisons, the power and moment of force patterns were normalized in time to $100 \%$ of the swing phase, ensemble averaged for each condition and for each subject separately, and then compared and interpreted with respect to the mechanical strategies employed by the subjects.

\section{Statistical Analyses}

Student's t-test and analysis of variance with repeated measures were used to compare the kinematic and kinetic data

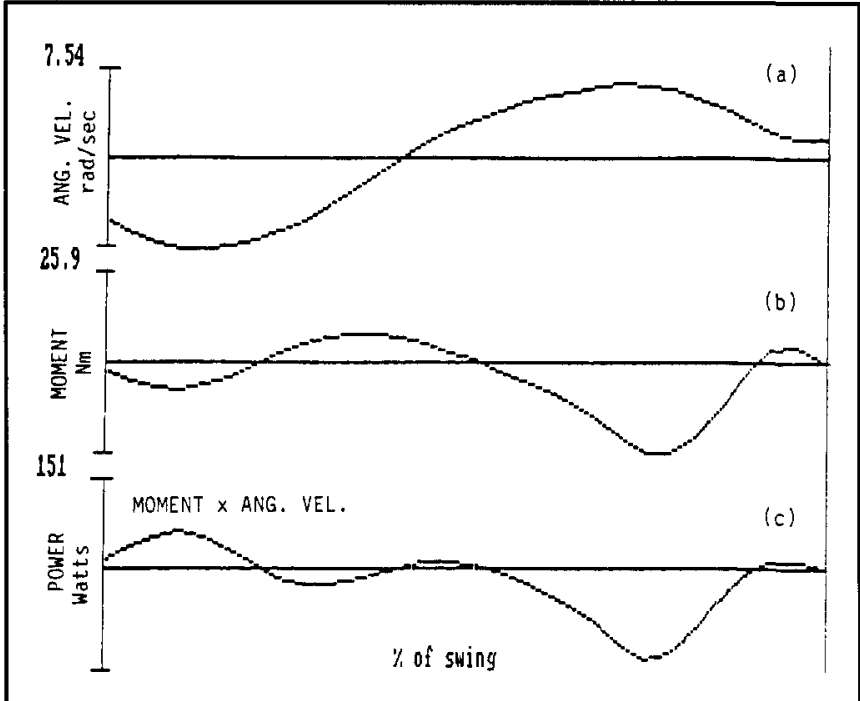

Figure I - Illustration of the joint angular velocity (a), moment of force (b) and their product, the resultant power (c) on one swing cycle of normal forward stepping at the knee joint. Positive and negative bursts in the moment of force curve $(b)$ correspond to extension and flexion moments respectively. Positive bursts in the mechanical power curve (c) indicate energy generation while negative bursts represent energy absorption. between the two groups and a significance level of $p=0.05$ was selected.

\section{Results}

Nine series of five consecutive forward and backward stepping motions (i.e., 90 trials per subject) were analysed. In this section we first consider the temporal organization of stepping (i.e., cadence), then we present the kinematic analysis, and finally we examine the joint kinetic results.

\section{Influence of Cadence}

The total duration of the stepping motions performed at natural cadence (a condition performed three times) varied across subjects and trials. As expected, patients with PD took, on the average, longer than control subjects to complete the task at natural cadence $[\mathrm{t}(14)=3.23, \mathrm{p}<0.003]$. However, for each cadence condition, all patients increased or decreased the duration of individual forward and backward stepping motions according to the instructions received (Table 1). Thus, instructions (i.e., cadence) had a significant effect on the duration of stepping $[\mathrm{F}(4,16)=52.33, \mathrm{p}<0.0001]$. Backward and forward motion durations were not statistically different $[F(1,16)=$ $0.341, p<0.57$ ]. Similarly, in the progressively accelerated cadence condition (i.e., from slow to fast), subjects on the average decreased their execution time between the first and last stepping motion in both forward and backward stepping motions indicating again that the instructions were understood and the task was executed accordingly.

\section{Joint Kinematics}

Maximum hip and knee flexion angles (Table 2) were influenced by cadence: the faster the cadence, the larger the flexion for the hip joint $[\mathrm{F}(2,16)=15.98, \mathrm{p}<0.0001]$ and the knee joint $[F(2,16)=28.08, p<0.0001]$. However, there was an interaction between subject groups and cadence for hip flexion $[F(2,32)=8.29, p<0.001]$ but not for knee flexion $[F) 2,32)=$ $0.45, \mathrm{p}=0.64]$. The interaction found at the hip joint is due to the fact that while there was an increased flexion angle with faster cadence in control subjects, this trend was not systematically observed in patients with $\mathrm{PD}$. In addition, hip joint flexion was significantly larger in patients with $\operatorname{PD}[F(1,16)=9.20, p<$ 0.008 ] while knee joint flexion was larger for control subjects although the relationship was not significant $[F(1,16)=4.36$, $p$ $<0.053$ ] as shown in Table 2.

Table 1: Averages (in Sec) of Individual Forward and Backward Stepping Motions for Five Conditions in Healthy Subjects and Patients With PD.

\begin{tabular}{lcccc}
\hline \hline & $\begin{array}{c}\text { Control Subjects } \\
\text { Forward }\end{array}$ & \multicolumn{2}{c}{ Patients W/PD } \\
& Backward & Forward & Backward \\
\hline +SLOW & 2.45 & 2.46 & 5.07 & 5.23 \\
SLOW & 1.79 & 1.84 & 2.89 & 3.49 \\
NC1 & 1.37 & 1.45 & 2.15 & 2.46 \\
FAST & 1.07 & 1.18 & 1.48 & 1.56 \\
+FAST & 0.79 & 0.98 & 1.27 & 1.39 \\
\hline
\end{tabular}

$(N C l=$ Natural Cadence $;$ Fast $=$ Faster Condition $;+$ Fast $=$ Fastest Condition; Slow $=$ Slower Condition $;$ and + Slow $=$ Slowest Condition). 
Under normal conditions, joint reversal from flexion to extension in stepping occurs first at the knee joint and then at the hip joint. In the present study, the hip and knee phase shift (Table 3) was significantly different between control subjects and patients with $\mathrm{PD}[\mathrm{F}(1,16)=9.51, \mathrm{p}<0.007]$ and across cadence conditions $[F(2,16)=12.56, p<0.0001]$. The difference was most obvious in the slow cadence condition. There was no significant differences in phase shift between forward and backward stepping motions $[F(1,16)=0.265, p=0.614]$ for control subjects and patients with PD. The differences observed indicate that in stepping patients with PD have larger phase shifts than control subjects.

Tangential velocity profiles of the metatarsal joint followed a biphasic pattern for control subjects and a monophasic pattern for patients with PD (Figure 2). This pattern was observed across cadences and subjects except for patient P3. However, we compared the step duration of P3 at the fastest cadence (Figure $2 \mathrm{~d})$ with that of a control subject at the fastest cadence. We observed that P3's tangential velocity profiles looked normal, that is biphasic (Figure $2 a, d$ ). Conversely, when step duration of a control subject at the slowest cadence (N2) is comparable to the step duration of a patient at the fastest cadence (P4), the observed velocity patterns of this control subject (N2) were

Table 2: Averages and Standard Deviations (in Degrees) of Maximum Hip Flexion (Above) and Knee Flexion (Below).

\begin{tabular}{|c|c|c|c|c|}
\hline & \multicolumn{4}{|c|}{ Hip Joint } \\
\hline & \multicolumn{2}{|c|}{ Control Subjects } & \multicolumn{2}{|c|}{ Patients W/PD } \\
\hline & Forward & Backward & Forwad & Backward \\
\hline+ Slow & $135.1(5.1)$ & $132.3(7.2)$ & $121.4(9.8)$ & $102.7(10.1)$ \\
\hline $\mathrm{NCl}$ & $131.2(6.0)$ & $127.4(5.5)$ & $122.5(4.5)$ & $120.4(5.1)$ \\
\hline +Fast & $123.9(4.7)$ & $129.8(5.4)$ & $120.3(5.5)$ & $118.7(7.8)$ \\
\hline
\end{tabular}

\begin{tabular}{|c|c|c|c|c|}
\hline & \multicolumn{4}{|c|}{ Knee Joint } \\
\hline & \multicolumn{2}{|c|}{ Control Subjects } & \multicolumn{2}{|c|}{ Patients W/PD } \\
\hline & Forward & Backward & Forward & Backward \\
\hline +Slow & $86.1(8.8)$ & $91.5(11.0)$ & $96.5(4.6)$ & $98.3(10.9)$ \\
\hline $\mathrm{NCl}$ & $82.9(9.7)$ & $87.6(10.9)$ & $93.1(4.6)$ & $91.0(6.5)$ \\
\hline +Fast & $80.1(7.5)$ & $84.1(9.2)$ & $91.3(5.8)$ & $88.6(10.5)$ \\
\hline
\end{tabular}

$($ NCI $=$ Natural Cadence $;+$ Fast $=$ Fastest Condition $;+$ Slow $=$ Slowest Condition). Because of Conventions Used in the Calculation of Joint Angles, Highest Values Correspond to Smallest Flexions.

Table 3: Phase Shift Between Knee and Hip Joint Reversal (in Sec) and Standard Deviations for Forward and Backward Stepping Motions for Three Conditions in Healthy Subjects and Patients With PD.

\begin{tabular}{lllll}
\hline & \multicolumn{2}{c}{ Control Subjects } & \multicolumn{2}{c}{ Patients W/PD } \\
& Forward & Backward & \multicolumn{1}{c}{ Forward } & \multicolumn{1}{c}{ Backward } \\
\hline +Slow & $0.14(0.77)$ & $-0.19(0.02)$ & $0.51(0.31)$ & $-0.67(0.59)$ \\
NCl & $0.10(0.22)$ & $-0.12(0.00)$ & $0.14(0.04)$ & $-0.17(0.05)$ \\
+Fast & $0.07(0.00)$ & $-0.07(0.01)$ & $0.11(0.02)$ & $-0.11(0.04)$ \\
Mean & $\mathrm{X}=0.10$ & $\mathrm{X}=0.11$ & $\mathrm{X}=0.25$ & $\mathrm{X}=0.32$ \\
\hline
\end{tabular}

$(N C I=$ Natural Cadence $;+$ Fast $=$ Fastest Condition $;$ and + Slow $=$ Slowest Condition). Values From the Hip Were Subtracted From Values From the Knee in Both Directions. Negative Values Indicate That Maximum Hip Flexion Was Attained Earlier Than Maximum Knee Flexion. mostly monophasic (Figure 2c). In the normal stepping cycle, the timing of the biphasic pattern occurs at the point of joint reversal at the knee. From our present results the role of this biphasic pattern at knee joint reversal is not clear, but what we can say is that when control subjects walk as slowly as patients with PD, they also show a monophasic pattern similar to that seen in patients with PD. Conversely (Figure 2), when patients with PD walk quickly, they show a "normal" biphasic pattern.

\section{Joint Kinetics}

In general, patients with PD did not perform like the control subjects. Some of the differences, however, were due to the fact that control subjects moved significantly faster than patients with PD for any given condition. Specifically, control subjects exhibited additional power bursts at mid-swing corresponding to energy absorption followed by generation from extensors to arrest and reverse the faster knee flexion produced in early swing (Figure 3).

However, some differences observed between the patients with PD and control subjects, were independent of movement speed. These coordination differences were most evident when patients with PD were compared to control subjects when taking velocity under consideration (i.e., comparing trials of similar duration as is done in Figures 4 and 5).

In forward stepping, extrapolation of the early swing data showed that control subjects began swing by generating hip flexor activity prior to lifting the foot off the ground (Figure 4). This hip activity was then soon diminished upon foot lift-off in coincidence with maximum knee flexor generation. With the decrease of this positive knee flexor power burst, hip power increased again to a second generation burst corresponding to the return of increased hip flexor influence. This triphasic coordination pattern of energy generation by the hip and knee flexors was accomplished within the first half of the forward swing phase and was exhibited by all control subjects in all cadence conditions although it was less evident in slower stepping conditions (Figure 4). The triphasic coordination pattern of hip/knee coordination was absent for patients with PD. Patients instead produced relatively simultaneous power generation bursts at both the hip and knee joints (Figure 4).

At the beginning of stepping backward, power profiles of control subjects and patients with PD were relatively similar for movements of comparable duration. Generally there was a generation burst by the hip flexors somewhat in advance of a generation burst by flexors at the knee (Figure 5). However, control subjects used hip flexors from the previous support phase to start limb lift-off and quickly switched to an extensor moment of the hip in early backward swing while patients with PD relied longer and more heavily on swing hip flexor activity (Figure 5). Thus, knee flexion became important at the beginning of backward swing for control subjects while it was further delayed in patients with PD.

Therefore, kinetic analysis of stepping motions of comparable cadences between patients with PD and control subjects revealed a number of differences in coordination patterns which also depended on movement direction. In the forward direction, patients with PD had no triphasic pattern between hip and knee flexor generation and in the backward direction their hip extensor moment was delayed (Figures 3,4 and 5). 


\section{Discussion}

The results presented above clearly indicate the presence of differences in the execution of repetitive stepping motions between control subjects and patients with PD. Although patients with $P D$ understand the instructions they receive and execute the stepping task adequately, they take longer to execute the sequence of stepping motions and their movement kinematics and kinetics reveal differences in coordination patterns.

A number of these differences are directly related to a reduced movement velocity in patients with PD. In the past, the presence of reduced movement velocity (bradykinesia) in Parkinson's disease has been considered as the primary symptom of basal ganglia dysfunction. ${ }^{14,15}$ In the present study the manifestation of this symptom is observed in the transition phase from flexion to extension. This phase is negotiated differently by the two groups of subjects. The lower movement veloc- ity of patients with PD does not require the intervention of knee extensor activity at midswing to break (i.e., absorb energy) and then reverse (i.e., generate energy) knee flexion. This reduced movement velocity at midswing also explains the differences observed in the tangential velocity profiles of the metatarsal joint in patients with PD (Figure 2) since when duration of step is taken under consideration this difference disappears. Recently, Sheridan \& Flowers ${ }^{9}$ have suggested that bradykinesia is not the primary symptom of PD. Rather it is the presence of endpoint variability in movement that constitutes the primary symptom and bradykinesia is only an adaptation, or a secondary symptom of the disease. One possible interpretation is that to maintain movement accuracy within acceptable limits, these patients slow their movement down to a level where they can use feedback to execute the movement adequately.

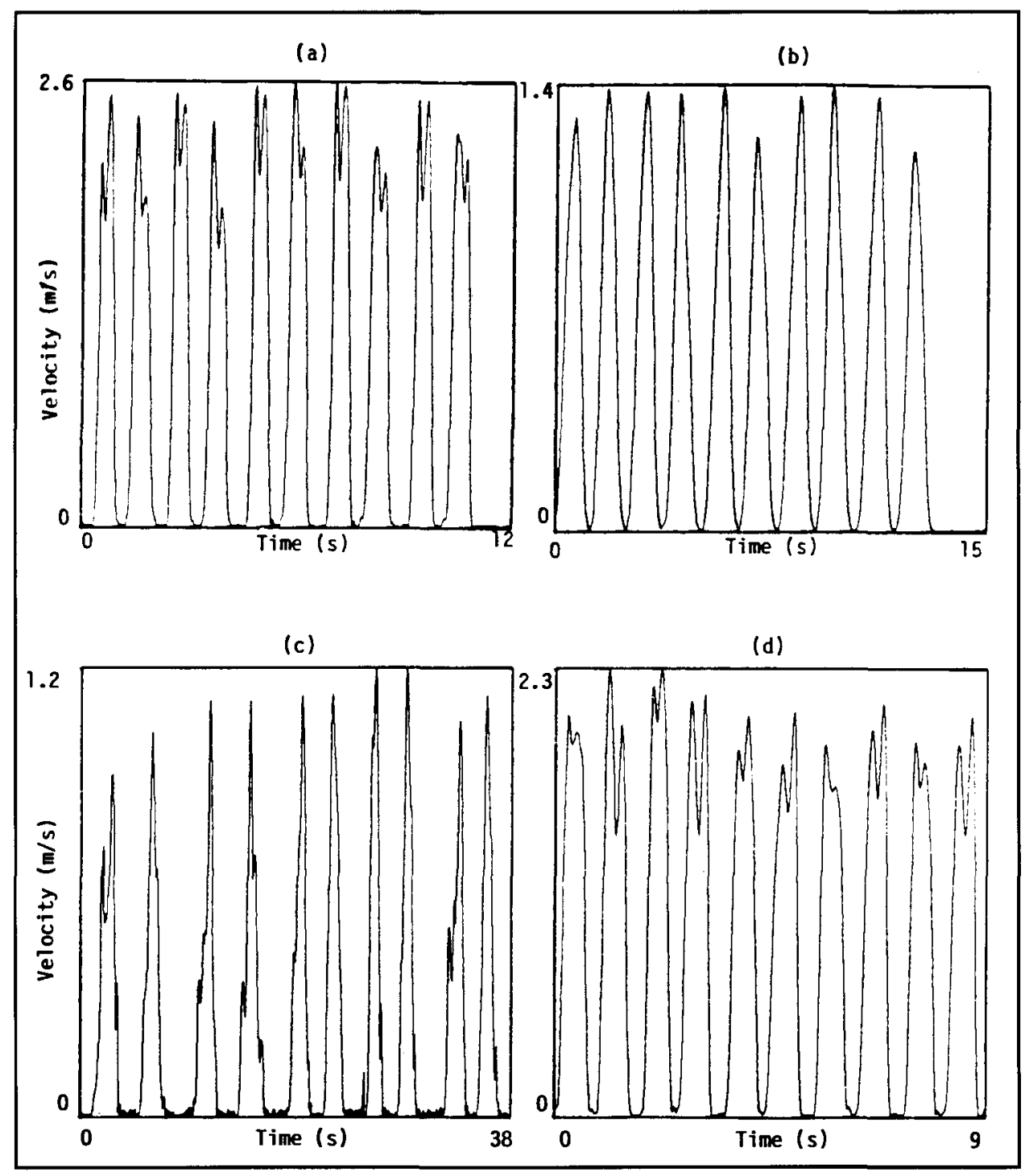

Figure 2 - Resultant velocity of metatarsal marker for five forward and five backward stepping motions for control subject $N 4(a)$ patient $P 4(b)$ and patient $P 3(d)$ in the fastest cadence condition and for $N 2$ at the slowest cadence (c), illustrating the biphasic ( $a$ and $d$ ) and monophasic ( $b$ and c) patterns of velocity. 


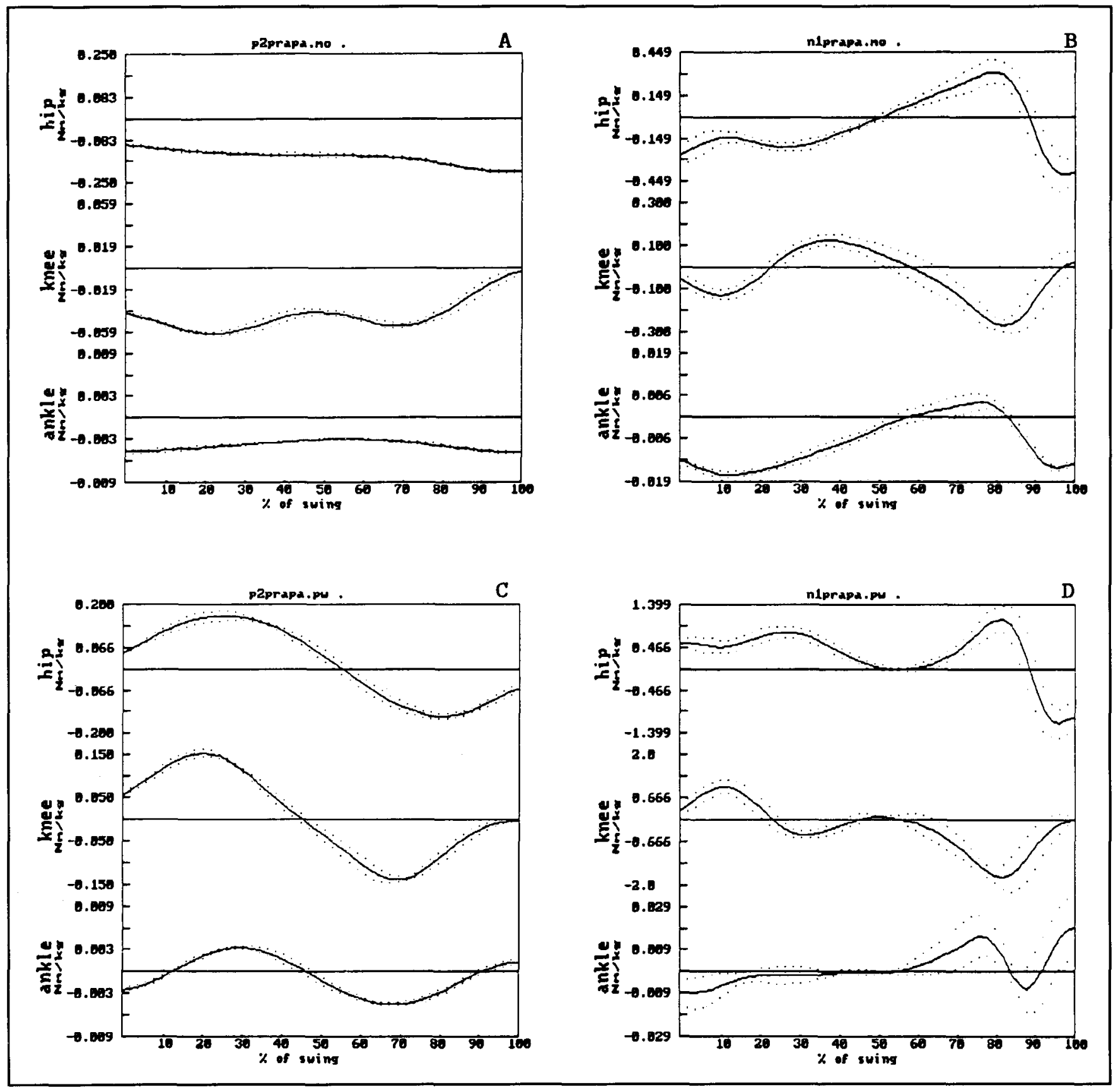

Figure 3 - Average joint moments of force and muscle powers on five forward stepping motions for patient with $P 2$ (a and c respectively) and control subject $N 1$ ( $b$ and d respectively) in the fastest cadence condition. Positive moments indicate predominant extensor activity. Positive power relates to net energy generation and negative power to net energy absorption. Moment and power curves must be interpreted in relation to joint kinematics (i.e., flexion and extension of the actual movement) in order to understand whether a particular joint action is actually contributing to the movement or slowing it down. Note the magnitude difference between control subjects and patients with PD. Dotted lines indicate plus and minus one standard deviation.

In the present study, joint actions in control subjects are active and anticipatory (Figure $3 \mathrm{~b}-$ hip and knee) while in patients with PD these joint actions are more passive and delayed (Figure 3a-hip and knee). As opposed to the active patterns of control subjects, patients with PD do not pull their limb down with the hip extensors at the end of forward swing, but rather, use hip flexor absorption. Going backward, patients with PD do not use the hip flexion from the previous support phase to anticipate limb lift-off. As a consequence they use less knee action at the beginning of swing. The absence of a triphasic pattern during power generation at the beginning of forward swing and the difference in joint action (hip/knee) to initiate backward stepping reveal the presence of coordination impairments which are not velocity-dependent. 


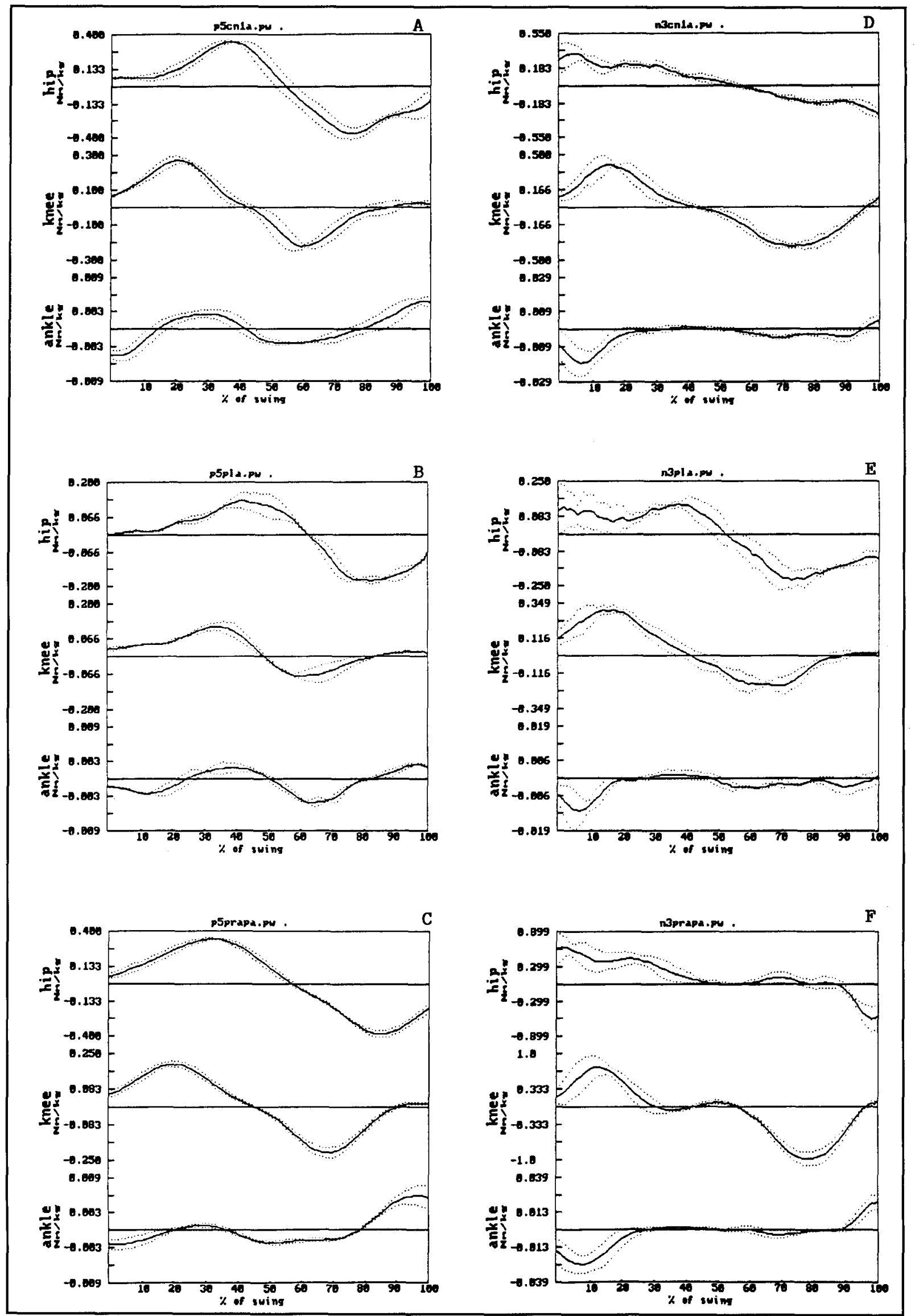

Figure 4 
Differences in power profiles are less clear in the backward than forward direction, suggesting that stepping backward (a rarely used task) is less affected than stepping forward (a more frequently used task). It is generally agreed that in visually guid- ed movements, humans tend to alternate between a feedback control strategy and an open-loop feedforward control strategy. ${ }^{16,17}$ According to Goldberg ${ }^{18}$ the predictive system (i.e., feedforward system) is located in the supplementary motor area

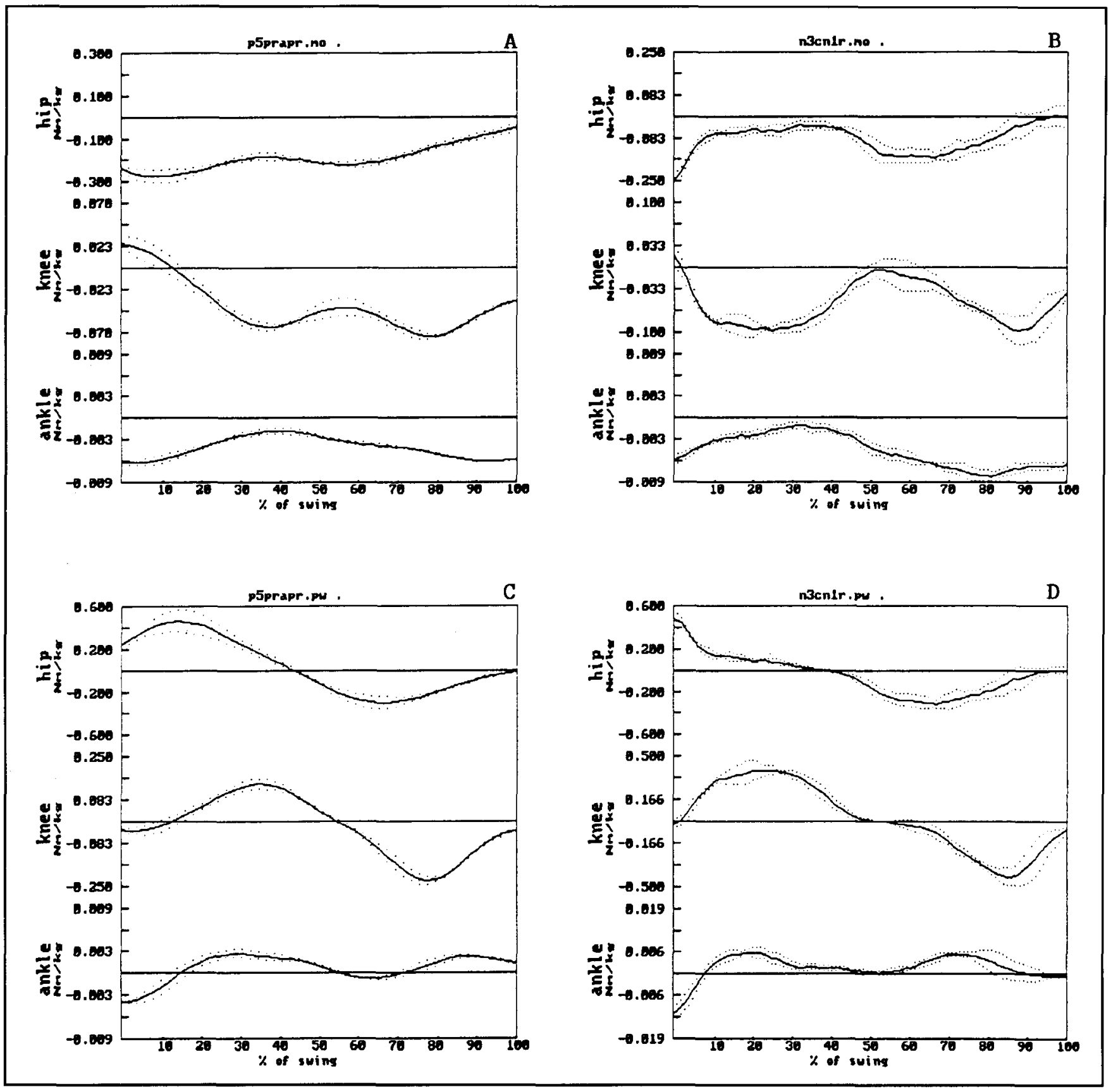

Figure 5 - Average joint moments of force and muscle powers on five backward motions for a patient with PD (a and c respectively) in the fastest condition and a control subject ( $b$ and d respectively) in the natural cadence condition. Positive moments indicate predominant extensor activity. Positive power relates to net energy generation and negative power to net energy absorption by the extensors or flexors for each joint. Note the magnitude difference between control subjects and patients with PD. Dotted lines indicate plus and minus one standard deviation.

Figure 4 (opposite) - Average muscle powers on five forward motions for a patient with $P D$ in the natural (a), slowest (b) and fastest (c) cadence condition and for a control subject (d, $e, f$ respectively). Positive power relates to net energy generation and negative power to net energy absorption indicated. Dotted lines indicate plus and minus one standard deviation. 
and receives its major input from the basal ganglia and the responsive system (i.e., feedback system) involves the premotor area and receives its major input from the sensory cortex (especially vision) and the cerebellum. It is known that patients with PD have difficulty recalling preprogrammed movement subroutines ${ }^{10}$ although in this study we noted that none of the patients with PD experienced freezing during the testing session. Thus, it seems logical that since the proactive/predictive mode of control (i.e., feedforward mode) is impaired, patients must rely more heavily on their reactive or responsive mode of control (i.e., feedback mode). Since stepping backward is as rarely used movement segment, we would expect that more feedback and less feedforward mechanisms are necessary to control its execution and this may explain why the differences between the two groups of subjects are smaller in the backward direction.

The phase shift between knee and hip joint reversals is markedly different in the slowest cadence condition for patients with PD (Table 3). In most subjects the difference or phase shift between knee and hip joint during stepping is about $0.10 \mathrm{~s}^{19}$ However, since control subjects are faster than patients, even at the slowest cadence, it is not possible at this point to know whether this change is velocity-dependent or reflects a different coordination pattern.

PD is not regarded as a perfect model of basal ganglia disease but it is generally considered as an adequate model especially during the early stages of the disease.

Despite the large amount of research investigating the contribution of the basal ganglia in the control of movement, results are still incomplete ${ }^{20-22}$ and according to Martin $^{23}$ the basal ganglia have been very obstinate in concealing their function. Changes in coordination patterns have been reported before in patients with PD during gait, ${ }^{8}$ in elbow movements ${ }^{24}$ and in speech. ${ }^{1,25}$ In the present study, however, we have shown that during complex movements, differences associated with bradykinesia disappear when velocity is accounted for. This was achieved by manipulating the cadence of stepping. By removing velocity dependent differences, we were able to unmask impairments in coordination patterns. The use of kinematic and especially kinetic analysis has allowed us to describe these differences in coordination in more detail.

\section{ACKNOWLEDGEMENTS}

We acknowledge the support of the Natural Sciences and Engineering Research Council (A1572) of Canada and we also gratefully acknowledge the participation of all patients.

\section{REFERENCES}

1. Connor NP, Abbs JH, Kelly JC, et al. Parkinsonian deficits in serial multiarticulate movements for speech. Brain 1989; 112: 9971009.

2. Johnels B, Ingvarsson PE, Thorselius $M$, et al. Disability profiles and objective quantitative assessment in Parkinson's disease. Acta Neurol Scand 1989; 79: 227-238.
3. Benecke JC, Rothwell C, Dick JPR, et al. Performance of simultaneous movements in patients with Parkinson's disease. Brain 1986; 109: 739-757.

4. Bloxham CA, Mindel TA, Frith CD. Initiation and execution of predictable and unpredictable movements in Parkinson's disease. Brain 1984; 107: 371-384.

5. Brown RG, Marsdsen CD. Visuospatial function in Parkinson's disease. Brain 1986; 109: 987-1002.

6. Sanes JN, Evarts EV. Psychomotor performance in Parkinson's disease. In: Delwaide PJ, Agnoli A, eds. Clinical Neurophysiology in Parkinsonism. Amsterdam and Oxford: Elsevier, 1985: 117 132.

7. Nutt G. Gait disorders. In: Jankovic J, Tolosa E, eds. Parkinson's Disease and Movement Disorders. Baltimore and Munich: Urban \& Schwarzenberg, Munich, 1988: 377-383.

8. Knutsson E. An analysis of parkinsonian gait. Brain 1972; 95: 475486.

9. Sheridan MR, Flowers KA. Movement variability and bradykinesia in Parkinson's disease. Brain 1990; 113: 1149-1161.

10. Marsden CD. Defects of movement in Parkinson's disease. In: Delwaide PJ, Agnoli A, eds. Clinical Neurophysiology in Parkinsonism. Amsterdam and Oxford: Elsevier, 1985: 107-115.

11. Hoehn MM, Yahr MD. Parkinsonism: onset, progression and mortality. Neurology 1967; 17: 427-442.

12. Bresler B, Frankel $\mathbf{J}$. The forces and moments in the leg during level walking. Trans ASME 1950; 72: 27-36.

13. McFadyen B. Anticipatory control for safe foot trajectories during obstructed walking. Ph.D. thesis, University of Waterloo, Ontario, 1988.

14. Denny-Brown D. Clinical symptomatology of diseases of the basal ganglia. In: Vinken PJ, Bruyn GW, eds. Diseases of Basal Ganglia. Amsterdam, North Holland: Handbook of Clinical Neurology, 1968; 6: 133-172.

15. Joubert M, Barbeau A. Akinesia in Parkinson's disease. In: Barbeau A, Brunette J-R, eds. Progress in Neurogenetics, International Congress Series, No. 175. Amsterdam: ExcerptaMedica; 1969; 366-376.

16. Miall RG, Weir DJ, Stein JF. Visuomotor tracking with delayed visual feedback. Neuroscience 1985; 16: 511-520.

17. Miall RG, Weir DJ, Stein JF. Manual tracking of visual targets by trained monkeys. Behav Brain Res 1986; 20: 185-201.

18. Goldberg F. Supplementary motor area structure and function: review and hypotheses. Behav Brain Sci 1985; 8: 567-616.

19. Beuter A, Carriere L, Boucher J. The role of biarticular muscles in stepping. In: deGroot G, Hollander AP, Huijing PA, et al., eds. Amsterdam: Free University Press, 1989: 43-47.

20. Marsden CD. Function of the basal ganglia as revealed by cognitive and motor disorders in Parkinson's disease. Can J Neurol Sci 1984: 11: 129-135.

21. Schneider JS, Lidsky TI. Basal Ganglia and Behavior: Sensory aspects of motor functioning, Hans Huber, Toronto, 1985.

22. Delwaide PJ, Agnoli A. Clinical Neurophysiology in Parkinsonism. Amsterdam: Elsevier Science Publications: 1985.

23. Martin JP. The Basal Ganglia and Posture. London: Pitman Medical Publishing Co.; 1967.

24. Hallett M, Shahani, BT, Young RR. Analysis of stereotyped voluntary movements at the elbow in patients with Parkinson's disease. J Neurol Neurosurg Psychiatry 1977; 40: 142-148.

25. Caruso AJ, Abbs JH, Gracco VL. Kinematic analysis of multiple movement coordination during speech in stutterers. Brain 1988; 111: 439-455. 